\title{
Evolução dos processos ecossistêmicos em reflorestamentos da Floresta Estacional Semidecídua ${ }^{1}$
}

\author{
Marcio Seiji Suganuma² e José Marcelo Domingues Torezan ${ }^{3,4}$
}

Recebido: 17.06.2013; aceito: 23.09.2013

\begin{abstract}
Evolution of ecosystem processes in Semideciduous Atlantic Forest restoration sites). Assessment for a successful ecological restoration can be done by comparisons with reference ecosystems (non degraded sites or habitat patches) selected in the borders of restoration sites. In this work a comparison among four reforestations (aging three to five years) and four neighboring of Semideciduous Atlantic Forest fragments were presented. Tree density in reforestations was near $50 \%$ of the average in forests, but necromass ranged from $24 \%$ to $33 \%$ and biomass from $18 \%$ to $75 \%$ of forest averages. High soil compaction ( $>2.0 \mathrm{Mpa}$ ) was observed only on younger reforestations, and compaction decreased root mass. Young Atlantic Forest restoration sites (up to five years) are important carbon sinks, but may have little influence on soil physical properties.
\end{abstract}

Key words: biomass, ecological restoration, necromass, soil compaction

RESUMO - (Evolução dos processos ecossistêmicos em reflorestamentos da Floresta Estacional Semidecídua). Para avaliar o sucesso na restauração ecológica é possível recorrer a comparações com ecossistemas de referência (áreas não degradadas ou fragmentos de habitat natural) selecionados em locais próximos. Neste trabalho comparamos aspectos do ecossistema em quatro reflorestamentos com espécies nativas (de três a cinco anos de implantação) adjacentes a quatro fragmentos de Floresta Estacional Semidecídua. Os reflorestamentos apresentaram em média 50\% da densidade de árvores dos fragmentos, mas a necromassa variou entre $24 \%$ e $33 \%$ e a biomassa entre $18 \%$ e $75 \%$ da média dos fragmentos. Compactação do solo considerada de alta resistência (>2,0 Mpa) foi encontrada apenas nos reflorestamentos de três e quatro anos, e a massa de raízes diminuiu com a compactação do solo. Reflorestamentos jovens (até cinco anos) são importantes para a captura de carbono atmosférico, mas podem ter pouco efeito sobre a compactação e a infiltração de água no solo.

Palavras-chave: biomassa, compactação do solo, necromassa, restauração ecológica

\section{Introdução}

Para avaliar o sucesso de técnicas de restauração de ambientes degradados, é preciso recorrer a protocolo cientificamente consistente (Ruiz-Jaén \& Aide 2005a), baseado em informações obtidas a partir de ecossistemas de referência (áreas que não foram degradadas ou fragmentos de habitat natural) e áreas-controle (i.e., áreas que não receberam manipulação visando restauração pela regeneração natural) selecionadas em sítios próximos e ambientalmente similares aos de restauração a serem avaliados (SER 2004, Clewell et al. 2005).
A avaliação do sucesso de iniciativas de restauração publicadas na literatura internacional está focada principalmente em medidas de biodiversidade, estrutura da vegetação e funcionamento do ecossistema (Ruiz-Jaén \& Aide 2005a, Ruiz-Jaén \& Aide 2005b), para que, segundo SER (2004), esses ecossistemas restaurados possam ter: 1) diversidade e estrutura de comunidades similares ao original; 2) espécies nativas da região; 3) grupos funcionais necessários para um desenvolvimento estável; 4) capacidade física necessária para a reprodução das populações ali presentes; 5) funções normais de desenvolvimento; 6) uma relação equilibrada com a paisagem onde

1. Parte da Dissertação de Mestrado do primeiro Autor

2. Universidade Estadual de Londrina, Pós-graduação em Ciências Biológicas

3. Universidade Estadual de Londrina, Laboratório de Biodiversidade e Restauração de Ecossistemas - LABRE, Caixa Postal 6001, 86057-970 Londrina, Paraná, Brasil

4. Autor para correspondência: torezan@uel.br 
está inserido; 7) resistência a potenciais distúrbios externos; 8) capacidade de suportar períodos de estresse; e 9) auto-sustentabilidade.

No âmbito global, em levantamento de todos os artigos publicados na revista Restoration Ecology (Vols. 1[1]-11[4]), Ruiz-Jaén \& Aide (2005a) avaliaram que o plantio de mudas e a semeadura direta são as técnicas mais utilizadas para a restauração ecológica (em mais de $80 \%$ dos casos), e que mais da metade dos artigos científicos que descrevem o sucesso da restauração utilizando essas duas técnicas foram produzidos na América do Norte (53\%), sendo somente três os estudos realizados na América do Sul (3\%). Então, há a necessidade de mais estudos que forneçam suporte técnico-científico para a legislação, na área de recuperação de florestas (Durigan et al. 2001), para a formulação de protocolo objetivo, para a avaliação do grau de sucesso dessas iniciativas de restauração ambiental, adaptado à realidade brasileira.

A análise de processos do ecossistema como, por exemplo, o ritmo de aumento de biomassa nas árvores e o acúmulo de serapilheira no solo, podem indicar uma recuperação mais rápida ou mais lenta de reflorestamentos ( $\mathrm{Lu}$ et al. 2003, Moreira \& Silva 2004). Assim, esses processos podem ser bons indicadores na comparação de reflorestamentos de diferentes idades, mostrando como está o andamento da restauração, usando os fragmentos florestais da região, como referência. A biomassa aérea tende a aumentar ao longo da sucessão secundária, de maneira mais rápida no início (Brown \& Salimon 2000) até atingir o clímax, quando a quantidade ganha e perdida entra em equilíbrio (Brown \& Lugo 1990, Engel \& Parrotta 2003). Assim, espera-se que a biomassa cresça com a idade em reflorestamentos jovens que não atingiram o clímax estrutural. Da mesma forma, a necromassa, composta principalmente pela serapilheira, deve aumentar com a idade dos reflorestamentos, o que indicaria o restabelecimento da ciclagem de nutrientes (Carvalho \& Vasconcelos 1999, Moreira \& Silva 2004), juntamente com uma recuperação das propriedades físicas do solo.

Dentro desse contexto político e científico, este estudo teve como objetivos comparar determinados processos do ecossistema entre reflorestamentos de diferentes idades, e entre esses reflorestamentos e ecossistemas de referência (fragmentos de Floresta Estacional Semidecídua), no norte do Estado do Paraná, para fornecer subsídios científicos para um protocolo de avaliação de sucesso de iniciativas de reflorestamento.

\section{Material e Métodos}

O estudo foi realizado no Estado do Paraná, Brasil, em quatro sítios, localizados nos municípios de Sertaneja, Alvorada do Sul, Primeiro de Maio e Rancho Alegre, em reflorestamentos de mata ciliar das margens do Reservatório de Capivara (rio Paranapanema, $22^{\circ} 47^{\prime} 45^{\prime \prime S}$ e $51^{\circ} 00^{\prime} 12^{\prime \prime} \mathrm{W}$, figura 1).

Cada sítio apresenta uma área de reflorestamento e um fragmento florestal natural adjacente, utilizado como referência. Os reflorestamentos apresentam diferentes idades, três anos, três anos e meio, quatro anos e cinco anos (tabela 1). Os fragmentos florestais são remanescentes de Floresta Estacional Semidecídua submontana, de diferentes tamanhos, circundados por matriz predominantemente agrícola. Todos os fragmentos sofreram corte seletivo de madeira em parte da sua área e apresentam trechos com predomínio de clareiras e emaranhados de cipós.

O clima da região é classificado como Cfa, zona tropical marginal com clima seco no inverno, sendo que o mês mais chuvoso do verão tem 10 vezes mais precipitação do que o mês mais seco de inverno; e a temperatura média do mês mais quente é de $22{ }^{\circ} \mathrm{C}$ (Maack 2002). Os solos da região são classificados como latossolo vermelho eutroférrico, destacando-se por ser de grande fertilidade natural (Stipp 2002). Por isso, o histórico do uso da terra em todos os sítios é de monoculturas, com rotatividade entre soja e milho.

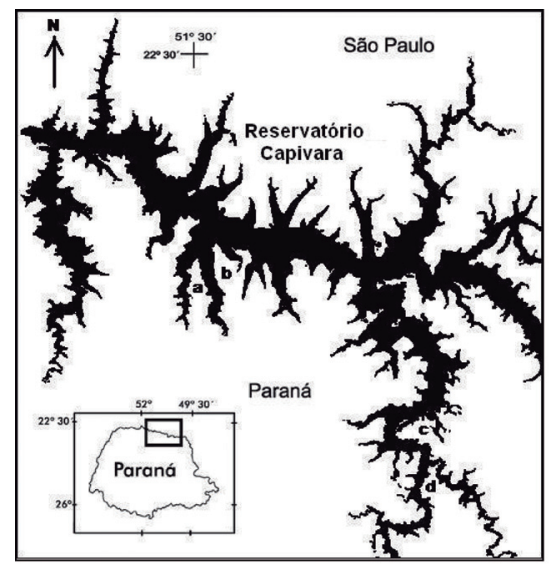

Figura 1. Reservatório de Capivara, no norte do Estado do Paraná, Brasil. As letras marcam os municípios de Alvorada do Sul (a), Primeiro de Maio (b), Sertaneja (c) e Rancho Alegre (d), onde estão localizados os reflorestamentos e os fragmentos florestais estudados.

Figure 1. Reservoir Capybara, in the north of Paraná State, Brazil. The letters indicates the municipality of Alvorada do Sul (a), Primeiro de Maio (b), Sertaneja (c) e Rancho Alegre (d), where the reforestation and forest fragments were studied. 
Os reflorestamentos foram realizados por meio de plantios mecanizados, em espaçamento $3 \times 2 \mathrm{~m}$, sem adubação, utilizando cerca de 40 espécies nativas da região, seguindo o modelo de Cavalheiro et al. (2002), com alta proporção de espécies pioneiras e secundárias iniciais, para o controle rápido de gramíneas invasoras por sombreamento.

Foram utilizadas parcelas de $5 \times 10 \mathrm{~m}$, alocando-se 20 parcelas em cada sítio, dez no reflorestamento e dez no fragmento florestal, totalizando 80 parcelas, distribuídas nas áreas de estudo. As parcelas foram alocadas de maneira a ficarem distribuídas em toda a área dos reflorestamentos. Nos fragmentos florestais foram evitadas, na medida do possível, as zonas afetadas pela extração madeireira e por superpopulação de cipós.

Foram registrados a altura total, o diâmetro na base (DB - $5 \mathrm{~cm}$ do solo) e o diâmetro a $1,30 \mathrm{~m}$ de altura do solo (DAP), para todos os indivíduos arbóreos dentro das parcelas, com DAP $\geq 2,5 \mathrm{~cm}$, para serem aplicados nas fórmulas de biomassa. Para os cálculos de biomassa aérea seca (BS), nos fragmentos e nos reflorestamentos, foram utilizadas, respectivamente, a equação para Floresta Semidecídua: $\mathrm{BS}=(0,523+\text { DAP. } 0,166)^{3}$ de Burguer \& Dellitti (1999) e as equações espécie-específica produzidas a partir de reflorestamentos no norte do Paraná por Jardim (2006).

Cada parcela foi dividida visualmente em quatro quadrantes iguais, no centro de cada quadrante foi coletado, com o auxílio de um quadrado de madeira, de $0,5 \times 0,5 \mathrm{~m}$, a serapilheira acumulada sobre o solo, tendo no final um total de $1 \mathrm{~m}^{2}$ de material coletado por parcela. As coletas de serapilheira foram feitas entre os meses de janeiro e abril. Todo material de cada parcela foi armazenado e posteriormente seco em estufa a $90{ }^{\circ} \mathrm{C}$ por dois dias, para posterior pesagem em balança semi-analítica.

Foram coletadas quatro amostras de solo com raízes por parcela, com coletor de solo de $10 \mathrm{~cm}$ de diâmetro e $5 \mathrm{~cm}$ de altura. Após secagem ao ar, o material foi peneirado em malha de $1 \mathrm{~mm}$ para remover o solo e outras partículas menores, e em seguida foi triado manualmente, visando separar a massa de raízes para pesagem em balança analítica. Os dados obtidos foram convertidos para $\mathrm{g} \mathrm{cm}^{-3}$.

A compactação do solo foi estimada por meio da medida da força, em MPa, necessária à penetração de uma ponta de prova, com um penetrômetro portátil de solo, segundo Soil Survey Division Staff (1993). Foram realizadas 20 medidas, distribuídas homogeneamente em cada parcela, utilizadas para o cálculo de uma média por parcela.

A permeabilidade do solo foi medida com um infiltrômetro adaptado, que consiste em um cano de metal graduado, com $20 \mathrm{~cm}$ de altura e $10 \mathrm{~cm}$ de diâmetro, que foi enterrado até $5 \mathrm{~cm}$ de profundidade, permanecendo $15 \mathrm{~cm}$ acima do solo. Em seguida, $500 \mathrm{ml}$ de água foram despejados para saturar de umidade a região superficial do solo. Após a absorção desse volume de água, foram despejados mais $500 \mathrm{ml}$ e cronometrado o tempo necessário para baixar cada $0,5 \mathrm{~cm}$ na coluna de líquido. Foram realizadas três repetições por parcela, evitando-se áreas próximas às árvores e aquelas compactadas pela rodagem de tratores.

Foram coletadas amostras da camada superficial do solo $(0-10 \mathrm{~cm})$ compostas de cinco perfurações em cada parcela, e as amostras foram enviadas para análise química de rotina (concentrações de P, C, $\mathrm{Ca}, \mathrm{Mg}$ e $\mathrm{K}$, soma de bases, $\mathrm{pH}$, saturação total de bases, matéria orgânica e granulometria) no Instituto Agronômico do Paraná (IAPAR).

Foram investigadas a homogeneidade de variâncias (teste de Levene) e a normalidade (teste de Kolmogorov-Smirnov), antes de comparar os dados entre os fragmentos e entre os reflorestamentos por meio de análise de variância (ANOVA), seguida de teste de Tukey $(\alpha=0,05)$. Para comparar os dados entre os reflorestamentos e os respectivos fragmentos de referência em um mesmo sítio, foi feito um teste- $t$ $(\alpha=0,05)$.

Tabela 1. Características dos sítios experimentais no Reservatório de Capivara, Estado do Paraná, Brasil.

Table 1. Characteristics of experimental sites in the Reservoir Capivara, Paraná State, Brazil.

\begin{tabular}{lccc}
\hline \multirow{2}{*}{ Município } & Fragmento & \multicolumn{2}{c}{ Reflorestamento } \\
\cline { 2 - 4 } & Área (ha) & Área (ha) & Idade (meses) \\
\hline Sertaneja & 33,3 & 32,1 & 36 \\
Alvorada do Sul & 11,3 & 128,1 & 42 \\
Primeiro de Maio & 38,2 & 43,3 & 48 \\
Rancho Alegre & 11,8 & 107,8 & 60 \\
\hline
\end{tabular}




\section{Resultados}

Foram amostrados 802 indivíduos arbóreos nos fragmentos e 404 nos reflorestamentos, o que representa a densidade média de 4.005 e 2.035 indivíduos ha $\mathrm{a}^{-1}$, respectivamente, com DAP igual ou maior que $2,5 \mathrm{~cm}$.

A média total de biomassa aérea seca dos quatro fragmentos florestais foi de $107 \mathrm{Mg} \mathrm{ha}^{-1}$, variando de $77 \mathrm{Mg} \mathrm{ha}^{-1}$ (P. de Maio) a $134 \mathrm{Mg} \mathrm{ha}^{-1}$ (R. Alegre), sem que houvesse diferença significativa entre os sítios (tabela 2). Nos reflorestamentos ocorreu um aumento significativo de biomassa aérea do mais novo para o mais velho, embora os reflorestamentos de idades próximas apresentassem biomassas iguais (tabela 2).

Comparando a biomassa aérea seca entre o fragmento e o reflorestamento de cada sítio, foram observadas diferenças entre os dois reflorestamentos mais novos, de três e três anos e meio, e os fragmentos de referência, com os reflorestamentos atingindo $16,5 \%$ e $28,5 \%$, respectivamente, da biomassa aérea das florestas nativas. Entre os dois reflorestamentos mais antigos (quatro e cinco anos) e os respectivos fragmentos adjacentes não houve diferença (tabela 2).

A média de massa de serapilheira dos fragmentos foi de 5,4 $\mathrm{Mg} \mathrm{ha}^{-1}$ (mín. de 4,8 e máx. de 6,4 $\mathrm{Mg} \mathrm{ha}^{-1}$ ) e não houve diferença entre os valores dos fragmentos florestais (tabela 3). Somente o reflorestamento mais antigo diferenciou-se dos demais reflorestamentos e do seu ecossistema de referência, no acúmulo de serapilheira (tabela 3 ).

A média geral de compactação do solo dos fragmentos foi de $0,35 \mathrm{MPa}$, e somente no fragmento de A. do Sul foi menor que os demais sítios (tabela 4).
Os reflorestamentos apresentaram um padrão em dois grupos separados, um grupo com Sertaneja (três anos) e P. de Maio (quatro anos) que apresentaram médias mais elevadas de compactação de solo; e outro grupo com os reflorestamentos de A. do Sul (três anos e meio) e R. Alegre (cinco anos) que apresentaram médias mais baixas. Comparando a compactação do solo entre o reflorestamento e o fragmento de referência de cada sítio, apenas no mais antigo não houve diferença significativa (tabela 4).

As médias da massa de raízes nos fragmentos não apresentaram diferenças, com uma média geral de $6,1 \mathrm{mg} \mathrm{cm}^{-3}$. As médias nos reflorestamentos foram sempre significativamente menores que as dos seus respectivos fragmentos, porém sendo estatisticamente diferentes apenas entre os reflorestamentos de idades extremas (tabela 5). Para os reflorestamentos, também foi feita uma correlação entre a massa de raízes e a compactação do solo, e o resultado foi significativo ( $\mathrm{n}=40, r=-0,35$ e $p=0,026)$.

A média de velocidade de descida da coluna de água no infiltrômetro nos fragmentos foi de $0,51 \mathrm{~mm} \mathrm{~s}^{-1}$ (mín. de 0,23 e máx. de $0,74 \mathrm{~mm} \mathrm{~s}^{-1}$ ). Nos reflorestamentos as médias de velocidade foram muito baixas, com 0,07 $\mathrm{mm} \mathrm{s}^{-1}$ em P. de Maio (quatro anos) e $0,03 \mathrm{~mm} \mathrm{~s}^{-1}$ em A. do Sul (três anos e meio). Nos sítios de Sertaneja (três anos) e R. Alegre (cinco anos) a permeabilidade foi praticamente nula, de acordo com a metodologia utilizada.

A granulometria do solo não diferiu entre o reflorestamento e o fragmento florestal de cada um dos sítios, mas variou entre os sítios. As comparações entre os reflorestamentos de diferentes idades e entre os fragmentos dos diferentes sítios apontaram

Tabela 2. Comparação das médias da biomassa aérea seca $\left(\mathrm{Mg} \mathrm{ha}^{-1}\right)$ : nas colunas, entre os fragmentos e entre os reflorestamentos nos diferentes sítios, indicada pelas letras maiúsculas; nas linhas, entre cada fragmento de referência e o reflorestamento correspondente, indicada pelas letras minúsculas. Reservatório de Capivara, Estado do Paraná, Brasil. Letras diferentes indicam que houve diferença significativa $(\alpha=0,05)$.

Table 2. Comparison of dry biomass $\left(\mathrm{Mg} \mathrm{ha}^{-1}\right)$ : between the fragments and reforestation among the various sites, are indicated by capital letters in the columns; the lines between each fragment reference and the correspondent reforestation are indicated by lowercase letters. Reservoir Capivara, Paraná State, Brazil. Different letters indicate significant difference $(\alpha=0.05)$.

\begin{tabular}{lccc}
\hline \multirow{2}{*}{ Sítios de estudo } & Fragmento & \multicolumn{2}{c}{ Reflorestamento } \\
\cline { 2 - 4 } & Biomassa $\left(\mathrm{Mg} \mathrm{ha}^{-1}\right)$ & Biomassa $\left(\mathrm{Mg} \mathrm{ha}^{-1}\right)$ & Idade (anos) \\
\hline Sertaneja & $115 \mathrm{Aa}$ & $19 \mathrm{Ab}$ & 3,0 \\
Alvorada do Sul & $102 \mathrm{Aa}$ & $29 \mathrm{ABb}$ & 3,5 \\
Primeiro de Maio & $77 \mathrm{Aa}$ & $60 \mathrm{BCa}$ & 4,0 \\
Rancho Alegre & $134 \mathrm{Aa}$ & $81 \mathrm{Ca}$ & 5,0 \\
\hline
\end{tabular}


a existência de dois grupos, onde Sertaneja e P. de Maio foram estatisticamente iguais, com solos mais arenosos, uma porcentagem mediana de argila e uma menor porcentagem de silte; e A. do Sul e R. Alegre formaram outro grupo, com porcentagens semelhantes de areia e argila e uma porcentagem baixa de silte (tabela 6). A variação na granulometria não se correlacionou com a variação na compactação.

A quantidade de carbono orgânico total do solo (C) não aumentou significativamente com a idade dos reflorestamentos $(p=0,28)$, apresentando valores similares em todos os locais (tabela 6). No entanto, todos apresentaram valores significativamente inferiores aos obtidos nos respectivos ecossistemas de referência. A razão da quantidade de C nos reflorestamentos pelo valor correspondente no fragmento florestal variou de 59,5\% (Sertaneja três anos) a 73,1\% (P. de Maio - quatro anos). Os valores de $\mathrm{pH}$ do solo seguiram um padrão similar ao do $\mathrm{C}$, com os reflorestamentos apresentando valores significativamente inferiores aos obtidos nos respectivos ecossistemas de referência (tabela 6). Quando comparados os valores de saturação de bases das áreas de cada sítio, somente o reflorestamento de quatro anos não apresentou diferença do seu fragmento florestal de referência. A matéria orgânica do solo nos reflorestamentos foi significativamente diferente entre todos eles e, ainda, cada reflorestamento diferiu de seu ecossistema de referência. Para os teores de minerais no solo não houve variações significativas (tabela 6). Não houve correlação entre variáveis químicas do solo e a biomassa, provavelmente em função da fertilidade excepcionalmente alta em todos os sítios.

\section{Discussão}

A média de biomassa aérea seca dos fragmentos florestais (107 Mg ha ${ }^{-1}$ ) é menor do que a encontrada por Burguer \& Dellitti (1999) numa Floresta Semidecídua do Estado de São Paulo (133,3 Mg ha ${ }^{-1}$ ). Esse fato pode ser explicado, em parte, pela menor densidade de indivíduos na região do Estado do Paraná (4.005 ind. ha-1) do que na de São Paulo (4.120 ind. ha-1), resultado de ações antrópicas como o corte seletivo de madeira (Araújo et al. 1999). Um fator adicional para explicar a biomassa menor nos fragmentos florestais seria o incremento da mortalidade de árvores em função de efeitos de borda, como aumento dos ventos, a super população de cipós e a combinação desses dois fatores (Rodrigues \& Nascimento 2006).

O rápido incremento de biomassa aérea nos reflorestamentos provavelmente guarda relação com a alta fertilidade do solo da região (Jardim 2006) e com o fato de os plantios terem sido feitos com espécies arbóreas de rápido crescimento (Lugo 1992, Burguer \& Dellitti 1999, Jardim 2006), mostrando a eficiência na incorporação de biomassa por reflorestamentos. Embora a fixação de carbono seja normalmente uma das metas da restauração ecológica (Engel \& Parotta 2003), os padrões observados apontam para o acúmulo de biomassa nos reflorestamentos superior aos valores dos fragmentos num prazo relativamente curto, o que suscita questões científicas interessantes sobre a estrutura e o funcionamento desses sistemas ecológicos recriados.

A massa de serapilheira acumulada sobre o solo nos fragmentos $\left(5,4 \mathrm{Mg} \mathrm{ha}^{-1}\right)$ foi maior do que a encontrada por Burguer \& Dellitti (1999) em um de

Tabela 3. Comparação das médias da massa de serapilheira acima do solo $\left(\mathrm{Mg} \mathrm{ha}^{-1}\right)$ : nas colunas, entre os fragmentos e entre os reflorestamentos nos diferentes sítios, indicada pelas letras maiúsculas; nas linhas, entre cada fragmento de referência e o reflorestamento correspondente, indicada pelas letras minúsculas. Reservatório de Capivara, Estado do Paraná, Brasil. Letras diferentes indicam que houve diferença significativa $(\alpha=0,05)$.

Table 3. Comparison of the means litter mass above ground $\left(\mathrm{Mg} \mathrm{ha}^{-1}\right)$ : columns among fragments and between the various sites reforestation are indicated by capital letters in the lines, between each fragment reference and the corresponding reforestation are indicated by lowercase letters. Reservoir Capivara, Paraná State, Brazil. Different letters indicate significant difference $(\alpha=0.05)$.

\begin{tabular}{lccc}
\hline \multirow{2}{*}{ Sítios de estudo } & Fragmento & \multicolumn{2}{c}{ Reflorestamento } \\
\cline { 2 - 4 } & Necromassa $\left(\mathrm{Mg} \mathrm{ha}^{-1}\right)$ & Necromassa $\left(\mathrm{Mg} \mathrm{ha}^{-1}\right)$ & Idade (anos) \\
\hline Sertaneja & $4,8 \mathrm{Aa}$ & $5,1 \mathrm{Aa}$ & 3,0 \\
Alvorada do Sul & $6,4 \mathrm{Aa}$ & $6,0 \mathrm{Aa}$ & 3,5 \\
Primeiro de Maio & $5,3 \mathrm{Aa}$ & $5,8 \mathrm{Aa}$ & 4,0 \\
Rancho Alegre & $5,1 \mathrm{Ab}$ & $7,2 \mathrm{Ba}$ & 5,0 \\
\hline
\end{tabular}


mata ciliar de Floresta Semidecídua inundável no Estado de São Paulo (3,2 $\mathrm{Mg} \mathrm{ha}^{-1}$ ). Essa diferença pode estar associada com a ocorrência de inundações, que removeriam os materiais depositados sobre o solo. Já nos reflorestamentos, o aumento da massa de serapilheira com a idade pode estar ligado à presença de muitas espécies pioneiras com folhas de vida curta (Ruiz-Jaén \& Aide 2005b), a desrama nos galhos mais baixos e à maior fragilidade dos reflorestamentos a danos físicos como chuvas e vendavais (Xiong \& Nilsson 1997).

No entanto, apesar da aparente correlação da massa de serapilheira com a idade do reflorestamento, e dada à semelhança, quanto a esse aspecto, entre o reflorestamento de cinco anos e seu ecossistema de referência, é possível sugerir que essa variável é adequada apenas para o monitoramento de estágios iniciais da restauração, não sendo esperadas mudanças expressivas na necromassa com idades maiores, se considerada apenas a serapilheira. Já galhos maiores e troncos de árvores mortas devem progressivamente ser incorporados, mas o estudo desses compartimentos exige técnicas especiais. $\mathrm{O}$ aumento na necromassa dos reflorestamentos muda as condições do solo e acelera o restabelecimento da ciclagem de nutrientes (Carvalho \& Vasconcelos 1999, Ruiz-Jaén \& Aide 2005b).

As médias de compactação do solo dos fragmentos florestais e dos reflorestamentos de três anos e meio e cinco anos podem ser classificadas como baixas; no entanto, os valores nos demais reflorestamentos foram maiores que 2,0 MPa, sendo assim, classificados como solos de alta compactação (Materechera et al. 1991, Soil Survey Division Staff 1993). Segundo Campbell et al. (1974) e Materechera et al. (1991), resistências à penetração acima de 2,0 MPa acarretam forte restrição física para o desenvolvimento das raízes. Apesar do reflorestamento mais antigo ser o único que não diferiu significativamente de seu ecossistema de referência, não há indício de que a evolução dos reflorestamentos

Tabela 4. Comparação das médias da compactação do solo (MPa): nas colunas, entre os fragmentos e entre os reflorestamentos nos diferentes sítios, indicada pelas letras maiúsculas; nas linhas, entre cada fragmento de referência e o reflorestamento correspondente, indicada pelas letras minúsculas. Reservatório de Capivara, Estado do Paraná, Brasil. Letras diferentes indicam que houve diferença significativa $(\alpha=0,05)$.

Table 4. Comparison of mean soil compaction (MPa): in the columns between the fragments and reforestation among the various sites are indicated by capital letters; the lines between each fragment and the corresponding reforestation reference are indicated by lowercase. Reservoir Capivara, Paraná State, Brazil. Different letters indicate significant difference $(\alpha=0.05)$.

\begin{tabular}{lccc}
\hline \multirow{2}{*}{ Sítios de estudo } & Fragmento & \multicolumn{2}{c}{ Reflorestamento } \\
\cline { 2 - 4 } & Compactação (MPa) & Compactação (MPa) & Idade (anos) \\
\hline Sertaneja & $0,37 \mathrm{Aa}$ & $2,13 \mathrm{Ab}$ & 3,0 \\
Alvorada do Sul & $0,12 \mathrm{Ba}$ & $0,50 \mathrm{Bb}$ & 3,5 \\
Primeiro de Maio & $0,49 \mathrm{Aa}$ & $2,16 \mathrm{Ab}$ & 4,0 \\
Rancho Alegre & $0,42 \mathrm{Aa}$ & $0,44 \mathrm{Ba}$ & 5,0 \\
\hline
\end{tabular}

Tabela 5. Comparação das médias da massa de raízes $\left(\mathrm{g} \mathrm{cm}^{-3}\right)$ : nas colunas, entre os fragmentos e entre os reflorestamentos nos diferentes sítios, indicada pelas letras maiúsculas; nas linhas, entre cada fragmento de referência e o reflorestamento correspondente, indicada pelas letras minúsculas. Reservatório de Capivara, Estado do Paraná, Brasil. Letras diferentes indicam que houve diferença significativa $(\alpha=0,05)$.

Table 5. Comparison of root mass average $\left(\mathrm{g} \mathrm{cm}^{-3}\right)$ : columns among fragments and between the various sites reforestation are indicated by the letters; in the lines, between each fragment and forestry corresponding reference are indicated by lowercase letters. Reservoir Capivara, Paraná State, Brazil. Different letters indicate significant difference $(\alpha=0.05)$.

\begin{tabular}{lccc}
\hline \multirow{2}{*}{ Sítios de estudo } & \multicolumn{2}{c}{ Fragmento } & \multicolumn{2}{c}{ Reflorestamento } \\
\cline { 2 - 4 } & Massa de raízes $\left(\mathrm{mg} \mathrm{cm}^{-3}\right)$ & Massa de raízes $\left(\mathrm{mg} \mathrm{cm}^{-3}\right)$ & Idade (anos) \\
\hline Sertaneja & $5,2 \mathrm{Aa}$ & $0,3 \mathrm{Ab}$ & 3,0 \\
Alvorada do Sul & $10,3 \mathrm{Aa}$ & $0,6 \mathrm{ABb}$ & 3,5 \\
Primeiro de Maio & $5,1 \mathrm{Aa}$ & $0,4 \mathrm{ABb}$ & 4,0 \\
Rancho Alegre & $3,9 \mathrm{Aa}$ & $0,6 \mathrm{Bb}$ & 5,0 \\
\hline
\end{tabular}


Tabela 6. Parâmetros químicos e físicos dos solos em cada um dos sítios, Reservatório de Capivara, Estado do Paraná, Brasil. Letra $\mathrm{F}$ representa as áreas dos fragmentos e a letra $\mathrm{R}$ as áreas dos reflorestamentos,

Table 6. Physical and chemical parameters of the soil in each site, Reservoir Capivara, Paraná State, Brazil. Letter F represents the fragments areas and $\mathrm{R}$ the reforestation areas.

\begin{tabular}{lrccccccc}
\hline \multirow{2}{*}{ Parâmetros do solo } & \multicolumn{3}{c}{ Sertaneja } & \multicolumn{3}{c}{ Alvorada do Sul } & \multicolumn{3}{c}{ Primeiro de Maio } & \multicolumn{3}{c}{ Rancho Alegre } \\
\cline { 2 - 9 } & $\mathrm{F}$ & $\mathrm{R}$ & $\mathrm{F}$ & $\mathrm{R}$ & $\mathrm{F}$ & $\mathrm{R}$ & $\mathrm{F}$ & $\mathrm{R}$ \\
\hline $\mathrm{P}\left(\mathrm{mg} \mathrm{dm}^{-3}\right)$ & 6,42 & 6,10 & 4,17 & 6,75 & 4,48 & 7,36 & 4,42 & 13,73 \\
$\mathrm{C}\left(\mathrm{g} \mathrm{dm}^{-3}\right)$ & 20,29 & 12,06 & 43,31 & 28,97 & 23,46 & 17,14 & 28,46 & 19,52 \\
$\mathrm{pH}$ & 6,08 & 5,10 & 6,74 & 5,73 & 5,95 & 5,32 & 6,59 & 5,95 \\
$\mathrm{Ca}\left(\mathrm{cmol}_{\mathrm{c}} \mathrm{dm}^{-3}\right)$ & 5,99 & 2,72 & 12,51 & 13,13 & 6,31 & 5,52 & 9,41 & 7,25 \\
$\mathrm{Mg}\left(\mathrm{cmol}_{\mathrm{c}} \mathrm{dm}^{-3}\right)$ & 1,99 & 1,47 & 3,56 & 3,37 & 2,48 & 2,13 & 2,86 & 1,92 \\
$\mathrm{~K}\left(\mathrm{cmol}_{\mathrm{c}} \mathrm{dm}^{-3}\right)$ & 0,23 & 0,15 & 0,77 & 0,74 & 0,42 & 0,42 & 0,36 & 0,60 \\
Soma de bases $\left(\mathrm{cmol}_{\mathrm{c}} \mathrm{dm}^{-3}\right)$ & 8,21 & 4,35 & 16,84 & 17,23 & 9,21 & 8,06 & 12,64 & 9,77 \\
Capacidade de troca de cátions $\left(\mathrm{cmol}_{\mathrm{c}} \mathrm{dm}^{-3}\right)$ & 10,93 & 7,59 & 19,47 & 21,62 & 12,19 & 12,75 & 15,27 & 13,00 \\
Saturação de bases $(\%)$ & 74,58 & 56,90 & 86,01 & 79,65 & 74,84 & 67,77 & 82,34 & 74,76 \\
Matéria orgânica (\%) & 29,84 & 20,18 & 78,77 & 61,66 & 44,39 & 39,99 & 46,05 & 33,73 \\
Argila (\%) & 20,7 & 21,0 & 63,0 & 61,0 & 38,7 & 53,7 & 46,7 & 39,3 \\
Silte $(\%)$ & 7,0 & 6,0 & 18,7 & 17,7 & 8,0 & 10,3 & 12,0 & 11,7 \\
Areia (\%) & 72,3 & 73,0 & 18,3 & 21,3 & 53,3 & 36,0 & 41,3 & 49,0 \\
\hline
\end{tabular}

está modificando a estrutura de compactação do solo nesse período inicial de desenvolvimento. Essa maior compactação do solo, nos reflorestamentos, favorece o desenvolvimento de espécies invasoras herbáceas (Bassett et al. 2005), que são, a princípio, controladas mecanicamente por capina e roçada e, posteriormente, pelo sombreamento do subosque.

No entanto, esse controle pode contribuir para retardar mudanças na compactação do solo, uma vez que a penetração de raízes está associada a mudanças na sua estrutura (Bassett et al. 2005, Ruiz-Jaén $\&$ Aide 2005b). Desse modo, mudanças no grau de compactação do solo possivelmente só serão observadas após um longo período, e na medida do desenvolvimento da vegetação natural no subosque do reflorestamento.

A massa de raízes finas nos fragmentos chegou a ser seis vezes maior que a dos reflorestamentos, refletindo, além da menor densidade de árvores, a dificuldade na penetração das raízes no solo compactado (Campbell et al. 1974, Materechera et al. 1991), corroborando as afirmações de Campbell et al. (1974), de que solos com menor resistência física proporcionam melhor ambiente para o desenvolvimento das raízes. Sendo assim, a estimativa da massa de raízes não foi um bom indicador para a avaliação das condições dos reflorestamentos, com a desvantagem adicional de ser um método bastante trabalhoso.
Comparando os dados de permeabilidade do solo entre os fragmentos e os reflorestamentos, estes não atingiram sequer $10 \%$ dos valores obtidos para os fragmentos, independente da variação na granulometria do solo. Como a compactação do solo, a infiltração de água possivelmente só apresentará variações nos reflorestamentos em um prazo maior, dados esses que vão contra a sugestão de Engel \& Parrotta (2003), que consideraram que essas variáveis devem se modificar em curto prazo. Sendo assim, a medição da permeabilidade do solo não é uma técnica aplicável na avaliação dos reflorestamentos nessa classe de idade (até cinco anos).

Todos os sítios apresentaram teores elevados de saturação de bases, para plantas cultivadas, de acordo com Tomé Jr. (1997). Como a saturação de bases sempre foi pouco menor nos reflorestamentos do que nos fragmentos florestais, e como não existem análises desses solos no passado, segundo Silva Jr. et al. (2005) pode-se supor que o tempo ainda não foi suficiente para a restauração desse aspecto da fertilidade dos solos nos reflorestamentos. Por outro lado, a média de concentração de fósforo no solo dos fragmentos florestais foi mais baixa do que os reflorestamentos, onde os teores de fósforo variaram de uma quantidade relativamente baixa no reflorestamento mais novo até a quantidade considerada suficiente no reflorestamento mais velho (igualmente, adotando-se padrões para plantas cultivadas, segundo Tomé Jr. 1997). Furtini 
Neto et al. (2000) afirmaram que o fósforo é essencial para o crescimento inicial das espécies pioneiras, o que explicaria ao menos em parte a elevada produção de biomassa nos reflorestamentos. No entanto, como não foi observada correlação entre a biomassa e o teor de $\mathrm{P}$, não é possível descartar a hipótese de que todos os teores, em reflorestamentos e fragmentos, sejam altos do ponto de vista da capacidade de captura de espécies arbóreas nativas.

\section{Agradecimentos}

À Duke Energy International, CNPq e Fundação Araucária, pelo apoio financeiro à pesquisa. M.S.S foi bolsista CAPES e J.M.D.T. contou com Bolsa produtividade do CNPq (processo 313854-2009-2). O apoio de Odair do Carmo Pavão, bolsista de Apoio Técnico do CNPq (processo 503836/2010-9) foi fundamental.

\section{Literatura citada}

Araújo, T.M., Higuchi N. \& Carvalho Jr., J.A. 1999. Comparison of formulae for biomass content determination in a tropical rain forest site in the state of Pará, Brazil. Forest Ecology and Management 117: 43-52.

Bassett, I.E., Simcock, R.C. \& Mitchell, N.D. 2005. Consequences of soil compaction for seedling establishment: Implications for natural regeneration and restoration. Austral Ecology 30: 827-833.

Brown, S. \& Salimon, C.I. 2000. Secondary forests in Western Amazonia: significant sinks for carbon released from deforestation? Interciencia 25: 198-201.

Brown, S. \& Lugo, A.E. 1990. Tropical secondary forests. Journal of Tropical Ecology 6: 1-32.

Burguer, D.M. \& Dellitti, W.B.C. 1999. Fitomassa epígea da mata ciliar do rio Mogi-Guaçu, Itapira - SP. Revista Brasileira de Botânica 22: 429-435.

Campbell, R.B., Reiscosky, D.C. \& Doty, C.W. 1974. Physical properties and tillage of paleudults in the Southern Coastal Plain. Soil Water Conservance 29: 220-224.

Carvalho, K.S. \& Vasconcelos, H.L. 1999. Forest fragmentation in central Amazonia and its effects on litter-dwelling ants. Biological Conservancy 91: 151-157.

Cavalheiro, A.L., Torezan, J.M.D. \& Fadelli, L. 2002. Recuperação de áreas degradadas: procurando por diversidade e funcionamento dos ecossistemas. In: M.E. Medri, E. Bianchini, O.A. Shibatta, \& J.A. Pimenta (eds.). A bacia do rio Tibagi. Londrina, pp. 213-224.
Clewell, A., Rieger, J. \& Munro, J. 2005. Guidelines for developing and managing ecological restoration projects, 2 ed. SER - Society for Ecological Restoration International. http://www.ser.org (acesso em 15.07.2007).

Durigan, G., Melo, A.C.G., Max, J.C.M., Vilas Boas, O. \& Contieri, W.A. 2001. Manual para recuperação de matas ciliares do oeste paulista. Páginas e Letras, São Paulo.

Engel, V.L. \& Parrotta, J.A. 2003. Definindo a restauração ecológica: tendências e perspectivas mundiais. In: P.Y. Kageyama, R.E. Oliveira, L.F.D. Moraes, V.L. Engel \& F.B. Gandara (eds.). Restauração Ecológica de Ecossistemas Naturais, Botucatu, pp. 1-26.

Furitni Neto, A.E., Siqueira, J.O., Curi, N., \& Moreira, F.M.S. 2000. Fertilização em reflorestamentos com espécies nativas. In: J.L.M. Gonçalves \& V. Benedetti (eds.). Nutrição e fertilização florestal, Piracicaba, pp. 351-383.

IPARDES - Instituto Paranaense de Desenvolvimento Econômico e Social. 1993. Cobertura florestal e consumo de madeira, lenha e carvão nas regiões de Londrina, Maringá e Paranavaí: subsídio para uma política florestal no estado do Paraná. IPARDES, Curitiba.

Jardim, P.S. 2006. Crescimento e biomassa de espécies arbóreas nativas da floresta estacional em reflorestamentos no norte do Paraná, Brasil. Dissertação de Mestrado, Universidade Estadual de Londrina, Londrina.

Kageyama, P., Gandara, F.B. \& Oliveira, R.E. 2003. Biodiversidade e restauração da floresta tropical. In: P.Y. Kageyama, R.E. Oliveira, L.F.D. Moraes, V.L. Engel \& F.B. Gandara (eds.). Restauração Ecológica de Ecossistemas Naturais, Botucatu, pp. 27-48.

Lamb, D., Parrota, J., Keenan, R. \& Tucker, N. 1997. Rejoing Habitat Remnants: Restoring Degraded Rainforest Lands. In: W.F. Laurance \& R.O. Biorregaard Jr. (eds.). Tropical Forest Remnants. The University of Chicago Press, Chicago, pp. 366-385.

Lu D., Mausel, P., Brondízio, E., \& Moran E. 2003. Classification of successional forest stages in the Brazilian Amazon basin. Forest Ecology and Management 181: 301312.

Lugo, A.E. 1992. Comparison of tropical tree plantations with secondary forests of similar age. Ecological Monographs 62: 1-41.

Maack, R. 2002. Geografia física do Estado do Paraná 3 ed. Imprensa Oficial, Curitiba.

Materechera, S.A., Dexter, A.R. \& Alston, A.M. 1991. Penetration of very strong soils by seedling roots of different plant species. Plant Soil 135: 31-41.

Mesquita, R.C.G., Ickes, K., Ganade, G. \& Willianson, G.B. 2001. Alternative successional pathways in the Amazon Basin. Journal of Ecology 89: 528-537. 
Moreira, P.R. \& Silva, O.A. 2004. Produção de serapilheira em área reflorestada. Revista Árvore 28: 49-59.

Rodrigues, P.J.F.P. \& Nascimento, M.T. 2006. Fragmentação florestal: breves considerações teóricas sobre efeito de borda. Rodriguésia 57: 63-74.

Ruiz-Jaen, M.C. \& Aide, T.M. 2005a. Restoration success: How is it being measured? Restoration Ecology 13: 569-577.

Ruiz-Jaen, M.C. \& Aide, T.M. 2005b. Vegetation structure, species diversity and ecosystem processes as measures of restoration success. Forest Ecology and Management 218: 159-173.

São Paulo - Secretaria do Meio Ambiente do Estado de São Paulo. 2007. Resoluções SMA 8, 21 e 47. http://www.ibot.sp.gov.br/legislacao.htm (acesso em 30.11.2006).

SER - Society for Ecological Restoration International Science and Policy Working Group. 2004. The SER primer in ecological restoration (Version 2). http://www. ser.org (acesso em 15.07.2007).
Silva Jr., W.M., Martins, S.V., da Silva, A.F. \& Marco Jr, P. 2005. Natural regeneration of trees and shrubs species in two sites of a tropical semideciduous forest, Viçosa, MG, Brazil. Scientia Florestalis 66: 169-179.

Soares, F.S. \& Medri, M.E. 2002. Alguns aspectos da colonização da bacia do rio Tibagi. In: M.E. Medri, E. Bianchini, O.A. Shibatta, \& J.A. Pimenta (eds.). A bacia do rio Tibagi. Universidade Estadual de Londrina, Londrina, pp. 103-107.

Soil Survey Division Staff. 1993. Soil survey manual. Department of Agriculture, Washington.

Stipp, N.A. 2002. Principais tipos de solo da bacia do rio Tibagi. In: M.E. Medri, E. Bianchini, O.A. Shibatta \& J.A. Pimenta (eds.). A bacia do rio Tibagi, Universidade de Londrina, Londrina, pp. 39-44.

Tomé Jr., J.B. 1997. Manual para interpretação de análise de solo. Agropecuária, Guaíba.

Xiong, S. \& Nilsson, C. 1997. Dynamics of leaf litter accumulation and its effects on riparian vegetation: a review. The Botanical Review 63: 240-264. 
